\title{
A Case Report of A Patient with Involvement of Six Cranial Nerves Without Any Sensory/Motor Weakness In Limbs With Cerebellar Signs Present in Very Short History with Multiple NCC Diagnosed on MRI Brain.
}

\author{
Dr. Bharat Veer Manchanda ${ }^{1}$,Dr. Amit Rai ${ }^{2}$,Dr. Rochak Pandey ${ }^{3}$, \\ Dr. Saloni Mehra ${ }^{4}$,Dr. Nishant Tayal ${ }^{5}$,Dr. ApoorvPratapSingh ${ }^{6}$ \\ ${ }^{1}$ Assi. Professor IndeptOf Medicine, Subharti Medical College, Meerut \\ ${ }^{2,3,5,6}$ Post Graduate In Dept Of Medicine, Subharti Medical College, Meerut \\ ${ }^{4}$ Post Graduate In Dept Of Surgery, Subharti Medical College, Meerut
}

\begin{abstract}
Graduate in Dept Of Surgery, Subharti Medical College, Meerut5- Post Graduate in Dept Of Medicine ,Subharti Medical College, Meerut6- Post Neurocysticercosis is the result of accidental ingestion of eggs of Taeniasolium (ie, pork tapeworm), usually due to contamination of food by people with taeniasis. In developing countries, neurocysticercosis is the most common parasitic disease of the nervous system and is the main cause of acquired epilepsy. In the United States, neurocysticercosis is mainly a disease of immigrants. We report here a case of involvement of six different cranial nerves $\left(I I^{\text {nd }}, V I^{\text {th }}, V I I^{\text {th }}, I X^{\text {th }}, X^{\text {th }}, X I^{\text {th }}\right)$ with cerebellar involvement and no sensory/motor involvement.
\end{abstract}

\section{Introduction}

Taeniasis is the intestinal infection of the adult tapeworm. When left untreated, a more serious condition known as cysticercosis develops as T. solium larvae invade body tissues. When larvae build up in the central nervous system, muscles, skin and eyes, it leads to neurocysticercosis - the most severe form of the disease and a common cause of seizures worldwide.

Clinical manifestations of neurocysticercosis vary with the locations of the lesions, the number of parasites, and the host's immune response.Many patients are asymptomatic. Possible symptomatic presentations include the following:

- Epilepsy: Most common presentation (70\%)

- Headache, dizziness

- Stroke

- Neuropsychiatric dysfunction

Abnormal physical findings, which occur in $20 \%$ or less of patients with neurocysticercosis, depend on where the cyst is located in the nervous system and include the following:

- Cognitive decline

- Dysarthria

- Extraocular movement palsy or paresis

- Hemiparesis or hemiplegia, which may be related to stroke, or Todd paralysis

- Hemisensory loss

- Movement disorders

- Hyper/hyporeflexia

- Gait disturbances

- Meningeal signs

The authors believe this case to constitute a medical rarity and thus worthy of being reported.

\section{Case Report}

A 60 year old,male patient presented to the Medicine OPD with the chief complaints of fever,blurring of vision,inability to move right eye laterally,facial deviation to left,unsteadiness,slurring of speech,difficulty in swallowing and in wearing clothes.Detailed history revealed that progression of symptoms to have occurred over a period of 15 days,starting with fever of low grade followed by other neurological symptoms.Pt. gave no history of Paraesthesias,numbness, weakness or weight loss. There was no bowel/bladder involvementand no past history of Diabetes Mellitus,Hypertension,Tuberculosis,CVA,Epilepsy,Out pt. was non-vegetarian by diet,chronic alcoholic and smoker.General examination was unremarkable.Detailed physical examination revealed blurring of vision,right lateral rectus palsy,facial deviation to left,loss of wrinkling over forehead on 
right side,loss of nasolabial fold on the left,weakness of muscles of facial expression,absence of gag reflex,difficulty in swallowing,deviation of uvula to the left and drooping of left shoulder.Patient had normal power (5/5) across all joints of all extremities,normal upper and lower limb tone,all deep tendon reflexes were intact and bilateral plantars were flexor.Pain,touch,temperature,joint position and proprioception senses were intact.Patient had staccato speech,dysdiadochokinesia (right upper limb>left), intention tremors,pastpointing,tandemwalking,Romberg's sign positive and heel shin test positive.MRI Brain was suggestive of multiple small to significant size oval nodular and at few places thick ring enhancing lesions scattered over bilateral cerebellum and upper brainstem diagnostic of Neurocysticercosis (NCC)

Based on clinical and radiological findings a diagnosis of Cerebellar NCC with involvement of Optic CN.,AbducensCN.,FacialCN.,Glossopharyngeal CN.,Vagus CN. And Spinal Accessory CN was established.

\section{Discussion}

Taeniasolium is the pork tapeworm belonging to cyclophyllidcestodes in the family Taeniidae. It is an intestinal zoonotic parasite found throughout the world, and is most prevalent in countries where pork is eaten. The adult worm is found in humans and has a flat, ribbon-like body, which is white in color and measures 2 to $3 \mathrm{~m}$ in length.

Humans are the only known definitive hosts for the T. solium. Human infection begins with the ingestion of infected raw or undercooked pork. The T. soliumlarvae gets digested out of the meat and attaches itself to the upper small intestine region. There it will mature and increase its number of proglottids. Terminal gravid proglottids will break off from the main body and will either pass out with the stool or worm its way out of the anus. In certain cases, 3 or 4 attached proglottids will pass out together. The eggs housed in the proglottids will be released and remain viable in the soil, sewage, and land for weeks. When pigs and humans ingest the eggs, the oncospheres will pierce through the intestinal walls, travel through the circulatory system, and plant itself in the subcutaneous and intramuscular tissues such as the brain and eyes. Cysticercosis will develop in these areas and will become invective in 9-10 weeks. Pigs will die in several months. In humans, cysticercosis has a variety of damaging effects on the central nervous system, vision, and brain functions.

In humans, autoinfection of T. solium eggs can occur by reverse peristalsis of the intesine.

The presence of T. solium worms in the human intestines usually does not cause major problems. Diarrhea, constipation, indigestion, and other mild stomach symptoms are common. Proglottids may also also crawl out of the anus.

The diagnosis in our patient was established on the basis of demonstration of Tenia larvae on stool sample microscopic examination,clinical signs and symptoms and MRI scan suggestive of extensive parasitic lesions of the cerebellum.

The case is unique in demonstrating simultaneous involvement of six cranial nerves along with extensive cerebellar involvement due to Teniasolium causing Neurocysticercosisand absolutely no sensorimotor or autonomic manifestations. 